\title{
Non-Gestational Ovarian Choriocarcinoma
}

National Cancer Institute

\section{Source}

National Cancer Institute. Non-Gestational Ovarian Choriocarcinoma. NCI Thesaurus.

Code C39991.

A malignant germ cell tumor that arises from the ovary and is composed of cytotrophoblasts, syncytiotrophoblasts, and extravillous trophoblasts. The prognosis is less favorable than gestational choriocarcinoma. 